\title{
Efficient Algorithms for Computing Sommerfeld Integral Tails
}

\author{
Ruzica Golubovic, Athanasios G. Polimeridis, and Juan R. Mosig, Fellow, IEEE
}

\begin{abstract}
Sommerfeld-integrals (SIs) are ubiquitous in the analysis of problems involving antennas and scatterers embedded in planar multilayered media. It is well known that the oscillating and slowly decaying nature of their integrands makes the numerical evaluation of the SI real-axis tail segment a very time consuming and computationally expensive task. Therefore, SI tails have to be specially treated. In this paper we compare two recently developed techniques for their efficient numerical evaluation. First, a partition-extrapolation method, in which the integration-then-summation procedure is combined with a new version of the weighted averages (WA) extrapolation technique, is summarized. The previous variants of WA technique are also discussed. Then, a review of double-exponential (DE) quadrature formulas for direct integration of the SI tails is presented. The efficient way of implementing the algorithms, their pros and cons, as well as comparisons of their performance are discussed in detail.
\end{abstract}

Index Terms-Double-exponential quadrature, extrapolation techniques, multilayered Green's functions, numerical analysis, Sommerfeld integrals, weighted averages algorithm.

\section{INTRODUCTION}

A NTENNAS and scatterers embedded in planar stratified media are frequently analyzed by means of Integral Equation (IE) formulations combined with a discretization procedure, like the Galerkin method of moments (MoM). The IE model can be cast in several variants, among which the mixed potential integral equation (MPIE) is usually considered to be more efficient because of its weakly-singular kernel [1]-[3]. The application of MoM for the solution of MPIE, basically involves two steps: first one is to fill up the MoM matrix, and the other one is to solve the matrix. In the process of filling the MoM matrix, Sommerfeld integrals (SIs) are introduced in the evaluation of the spatial-domain Green's functions (GFs) from their spectral-domain counterparts

$$
S_{\nu}\left\{\tilde{G}\left(k_{\rho} ; z \mid z^{\prime}\right)\right\}=\frac{1}{2 \pi} \int_{0}^{\infty} \tilde{G}\left(k_{\rho} ; z \mid z^{\prime}\right) \mathrm{J}_{\nu}\left(k_{\rho} \rho\right) k_{\rho} d k_{\rho} .
$$

Here, $\tilde{G}$ is the spectral domain Green's function of the generic layered media shown in Fig. 1, $\mathrm{J}_{\nu}$ is the Bessel function of the first kind of order $\nu, \rho$ is the horizontal distance between the

Manuscript received April 08, 2011; revised October 08, 2011; accepted December 02, 2011. Date of publication March 01, 2012; date of current version May 01, 2012. This work was supported in part by the European COST Action IC0603 "ASSIST".

The authors are with the Laboratory of Electromagnetics and Acoustics (LEMA), Ecole Polytechnique Fédérale de Lausanne (EPFL), CH-1015 Lausanne, Switzerland (e-mail: ruzica.golubovic@epfl.ch; athanasios.polymeridis@epfl.ch; juan.mosig@epfl.ch).

Color versions of one or more of the figures in this paper are available online at http://ieeexplore.ieee.org.

Digital Object Identifier 10.1109/TAP.2012.2189718

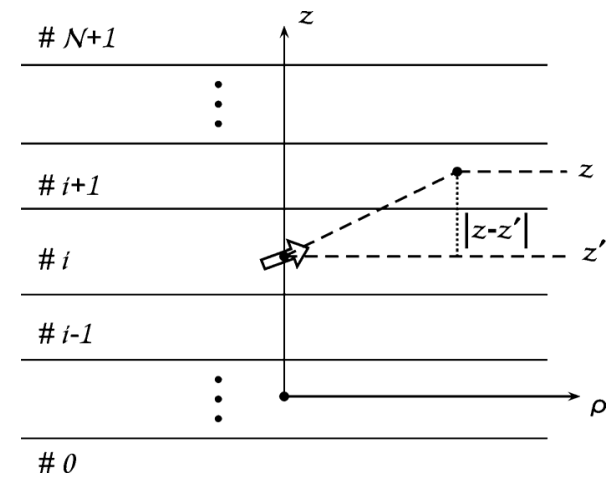

Fig. 1. Generic stratified media showing a point source (level $z^{\prime}$ ) and a field observation point (level $z$ ) separated by a radial distance $\rho$. The medium can be terminated or not by PEC, PMC and impedance planes.

field and source points, and $z, z^{\prime}$ are the vertical coordinates of those points, respectively.

The oscillatory and slowly decaying kernels of the SIs, as well as the occurrence of singularities on and/or near the integration path in the complex $k_{\rho}$-plane, make computation of the SIs very difficult and time-consuming. Having in mind that SIs need to be repeatedly evaluated in the process of filling the MoM matrix, it is clear that, in the case of objects placed in multilayered media, filling the MoM matrix might take the dominant part of the computational load involved in the solution of the MPIE via MoM. Therefore, efficient and fast computation of SIs is of paramount relevance.

The problem of efficient evaluation of the SIs, although nowadays a classic one, it is still attracting a lot of attention. The numerous publications on this topic can be mainly categorized in two classes. In the first class, SIs are approximated by closed-form analytical formulas (see [4]-[11] among others). A typical example is provided by the "complex images" formulations. There, spectral-domain GFs are represented as finite sums of special functions which admit analytical solution. These methods result in very fast algorithms and are becoming increasingly popular. But, there will be always some uncertainties about their range and precision. In a very recent paper dealing with closed-from GFs [11] it was pointed out the importance of alternative "safer" schemes that can be always used as benchmarks for testing purposes or for fine-tuning the coefficients of an analytical approximation. These alternative strategies are provided by a pure numerical evaluation of the SIs. Indeed if the numerical algorithms are efficient enough, this second class of methods, can also be used per se as a part of a complete software tool implementing the IE-MoM model. The main assets of these sophisticated numerical methods are highly accurate results and error controllable behavior. The 
most known representative is the integration-then-summation method combined with one of the numerous extrapolation techniques, such as weighted averages (WA) method, Shanks or generalized Levin transformation, among others [12], [13]. Also, several techniques for the direct integration of SI tails exist [14]-[18].

The aim of this paper is to discuss two recently developed techniques for the efficient numerical evaluation of SI tails, with the target of assessing the advantages and disadvantages of their application. These two techniques are a new version of the weighted averages (WA) algorithm and a specially tailored double exponential (DE) quadrature formula.

WA method was originally introduced in the early eighties by Mosig [19]-[21]. It was later further extended and improved in a very complete paper by Michalski [13], who found WA to be a sophisticated version of the Euler transformation. Since then it has been widely used in the EM community. The method has been proven to be one of the most efficient extrapolation methods for the convergence acceleration of the sequences obtained when the integration-then-summation technique is applied to SIs. In this paper we use the classic Mosig-Michalski version but also a very interesting and recently introduced new version of WA [22].

The double exponential (DE) quadrature formula is a powerful numerical tool proposed by Takahashi and Mori in 1974 for the integration of the functions with singularities at the endpoints of the integration interval [23]. It is based on a change of variables that guarantees a DE decay of the integrand. Unfortunately, the original formula was not efficient in computing slowly decaying oscillatory functions over $(0, \infty)$. Hence, to overcome this weakness, Ooura and Mori proposed a robust DE formula for Fourier-type integrals [24]. The key idea of the new transformation was slightly different: the nodes of the new quadrature approach rapidly (double exponentially) the zeros of sine/cosine function, thus allowing computation of the Fouriertype integrals with a small number of function evaluations. A variant with Bessel function zeros as nodes was proposed in [25]-[27]. Finally, following similar ideas, a very efficient tool for the evaluation of semi-infinite integrals with Bessel function kernels was suggested in [28] and then adapted to the SI tails in [18] by the authors.

This paper is organized as follows. A brief overview of the WA and DE methods is presented in Section II. The efficiencies of the methods are compared throughout a series of numerical examples in Section III, with a special attention to the efficient way of implementing the algorithms. Finally, some conclusions and recommendations are drawn in Section IV.

\section{SOMMERFELD INTEGRAL TAILS}

Among various integration paths that can be employed for the evaluation of the SIs (1), the real-axis path intended into the first quadrant (see Fig. 2) for avoiding branch points and pole singularities has been proven to be most convenient for multilayered media [29], since it obviates the need to extract the poles.

There are mainly two difficulties arising when computing SIs. The first one is related to the possible presence of singularities in the integrand. The second difficulty is due to the oscillating

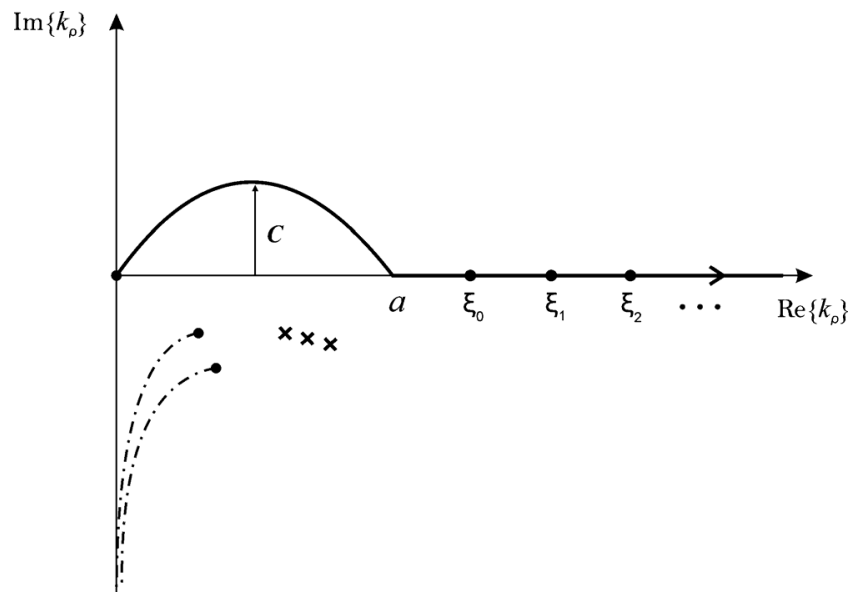

Fig. 2. Deformed integration path for the computation of Sommerfeld integrals.

integrand which converges slowly, or even diverges, and which has to be considered over an infinite interval. Luckily enough, these two problems can be always treated separately, by adequately splitting the integral into two parts $\left(0, \xi_{0}\right)$ and $\left(\xi_{0}, \infty\right)$

$$
S_{\nu}=\frac{1}{2 \pi}\left(I_{\nu}+T_{\nu}\right)
$$

The first part of the integral, $I_{\nu}$, joins the origin to the first break point $\xi_{0}>a$, where $a$ is appropriately selected in order to ensure that the remaining integral, $\operatorname{Re}\left\{k_{\rho}\right\}>a$ is free of singularities. Although techniques for adaptively selecting the integration path and numerically evaluating $I_{\nu}$ are out of the scope of this paper, the approach followed here inspired from [29] is briefly recalled for the sake of completeness. The first part of the integral is again split into two parts.

$$
I_{\nu}=\frac{1}{2 \pi}\left(I_{\nu_{a}}+I_{\nu_{b}}\right)
$$

$I_{\nu_{a}}$ is the integral over the interval $(0, a)$. Its path is deformed into the first quadrant and the exact shape is not important as far as the singularities are circled around and we are at the proper Riemann sheet. Here we have used a half-sine shape, but other contours (for example semi-elliptical [30]) could be used as well. The following parameters are selected: $a=k_{0}\left(\sqrt{n_{\max }}+\right.$ 1 ), where $n_{\max }=\max \left\{\operatorname{Re}\left\{\epsilon_{r_{i}} \mu_{r_{i}}\right\}\right\}, i=0,1, \ldots, N+1$ (see Fig. 1), and the maximum sine height $c$ is limited by the exponential growth of the Bessel function when $k_{\rho}$ becomes complex and is set to

$$
\frac{c}{k_{0}}= \begin{cases}\min \left(1, \frac{1}{k_{0} \rho}\right), & \text { if } \rho>\operatorname{Re}\left\{\left\{\left|z-z^{\prime}\right|\right\}\right\} \\ 1, & \text { otherwise. }\end{cases}
$$

$I_{\nu_{b}}$ is the real-axis integral from $a$ to the first break point $\xi_{0}>a$. Exact zero crossings, extremum points and asymptotic half-periods of Bessel functions are common choices of break points [13]. Both integrals, $I_{\nu_{a}}$ and $I_{\nu_{b}}$, are computed by an adaptive quadrature based on the Paterson rule [31]. The remaining integral

$$
T_{\nu}=\int_{\xi_{0}}^{\infty} \tilde{G}\left(k_{\rho} ; z \mid z^{\prime}\right) \mathrm{J}_{\nu}\left(k_{\rho} \rho\right) k_{\rho} d k_{\rho}
$$


is the semi-infinite SI tail on the real axis. Although it is free of singularities, it calls for a special treatment because of its oscillatory and slowly decaying integrand. Below, we discuss two recently developed methods for the fast and accurate numerical evaluation of the SI tails (5).

\section{A. Partition-Extrapolation Method Involving WA Technique}

Partition-extrapolation procedure stands for the traditional and most popular approach for calculating SI tails. Indeed, applying the integration-then-summation procedure, the SI tail is transformed into an infinite sum of partial integrals over the finite subintervals

$$
\begin{aligned}
T_{\nu} & =\sum_{i=0}^{\infty} \int_{\xi_{i}}^{\xi_{i+1}} \tilde{G}\left(k_{\rho} ; z \mid z^{\prime}\right) \mathrm{J}_{\nu}\left(k_{\rho} \rho\right) k_{\rho} d k_{\rho} \\
& =\sum_{i=0}^{\infty} u_{\nu i} .
\end{aligned}
$$

The conventional approach for the evaluation of (6) consists in finding the limit of the sequence of partial sums

$$
T_{\nu}=\lim _{n \rightarrow \infty} T_{\nu n}=\lim _{n \rightarrow \infty} \sum_{i=0}^{n} u_{\nu i} .
$$

As this sequence converges extremely slowly, i.e., remainders $r_{n}=T_{\nu n}-T_{\nu}$ do not decay rapidly as $n \rightarrow \infty$, in order to achieve high accuracy via direct sum we need to evaluate extremely large number of partial integrals, $u_{\nu i}$, which is unacceptable in terms of computational cost. Instead, application of one of the extrapolation techniques allows us to infer the limit value in (7) by calculating just a few partial integrals $u_{\nu i}$. As mentioned in the introduction, WA is a generalization of the Euler transformation, which instead of simple means uses weighted means of consecutive partial sums

$$
T_{\nu n}^{\prime}=\frac{W_{n} T_{\nu n}+W_{n+1} T_{\nu n+1}}{W_{n}+W_{n+1}}
$$

where $W_{n}$ are the weights to be chosen. Since $T_{\nu n}^{\prime}=T_{\nu}+r_{n}^{\prime}$, (8) can be written in a following form:

$$
T_{\nu n}^{\prime}=T_{\nu}+\underbrace{\frac{W_{n} r_{n}+W_{n+1} r_{n+1}}{W_{n}+W_{n+1}}}_{r_{n}^{\prime}} .
$$

Obviously, the optimal solution would come from the annihilation of the remainders $r_{n}^{\prime}$ of the linearly transformed sequence by imposing an appropriate ratio of the weights

$$
\eta=\frac{W_{n+1}}{W_{n}}=-\frac{r_{n}}{r_{n+1}} .
$$

In this point the WA method could be considered complete if the remainders $r_{n}$ were explicitly known, which is, unfortunately, not the case for the sequences of our interest. Instead, we use their asymptotic expansion

$$
r_{n}=\omega_{n} \sum_{i=0}^{\infty} a_{i} \xi_{n}^{-i}, \quad n \rightarrow \infty
$$

where $\omega_{n}$ are remainder estimates and $a_{i}$ are the associated coefficients, as given in [13]. Based on the asymptotic behavior of the spectral domain GFs

$$
\tilde{G}\left(k_{\rho} ; z \mid z^{\prime}\right) \sim \frac{e^{-k_{\rho}\left|z-z^{\prime}\right|}}{k_{\rho}^{\mu}}\left(C+O\left(k_{\rho}^{-1}\right)\right)
$$

and using equidistant break points separated by asymptotic halfperiods of Bessel function, $\chi=\pi / \rho$, as limits of partial integrals $u_{\nu i}$, the following analytical expression for the remainder estimates is obtained

$$
w_{n}=(-1)^{n+1} e^{-n \cdot \chi\left|z-z^{\prime}\right|} \xi_{n}^{q},
$$

for $\rho>0$, where $q=0.5-\mu$. In special cases, when $\rho=0$, the interval length $\chi=\pi /\left|z-z^{\prime}\right|$ is suggested in [13] for the partial integrals $u_{\nu i}$, and accordingly the $(-1)^{n+1}$ factor in (13) vanishes. Finally, incorporating these remainder estimates into the WA transformation (8), the following recursive WA scheme is obtained

$$
T_{\nu n}^{l+1}=\frac{T_{\nu n}^{(l)}+\eta_{n}^{(l)} T_{\nu n}^{(l)}}{1+\eta_{n}^{(l)}}, \quad n, l \geq 0
$$

with

$$
\begin{aligned}
\eta_{n}^{(l)} & = \pm e^{\chi\left|z-z^{\prime}\right|}\left(\frac{\xi_{n+1}}{\xi_{n}}\right)^{-q+2 l} \\
& \approx \pm e^{\chi\left|z-z^{\prime}\right|}\left(1+\frac{-q+2 \cdot l}{\vartheta+n}\right),
\end{aligned}
$$

where the plus and minus sign apply to $\rho>0$ and $\rho=0$ cases, respectively and $\vartheta=\xi_{0} \rho / \pi$. The aforementioned recursion can be represented by a triangular scheme

$$
\begin{array}{lllll}
T_{\nu_{0}}^{(0)} & T_{\nu_{0}}^{(1)} & \ldots \ldots \ldots \ldots & T_{\nu_{0}}^{(l)} \\
T_{\nu_{1}}^{(0)} & T_{\nu_{1}}^{(1)} & \ldots & T_{\nu_{1}}^{(l-1)} & \\
T_{\nu_{2}}^{(0)} & \ldots & T_{\nu_{2}}^{(l-2)} \\
\vdots & & & \\
T_{\nu_{l}}^{(0)} & & &
\end{array}
$$

where in the first column $T_{\nu n}^{(0)}=T_{\nu n}$ and $T_{\nu 0}^{(l)}$ is the best approximation of $T_{\nu}$, given the partial sums $T_{\nu 0}, \ldots, T_{\nu l}$.

In the original version of the WA method [19], the remainders $r_{n}$ are expanded into infinite series using integration by parts, which is truncated for numerical purposes

$$
\begin{aligned}
r_{n} & =T_{\nu}-T_{\nu n} \\
& =\operatorname{Re}\left\{e^{-\gamma \xi_{n}} \sum_{k=1}^{n}\left[\frac{\partial^{k-1}}{\partial k_{\rho}^{k}}\left(k_{\rho}^{q}\right)\right]_{\left.\right|_{k_{\rho}=\xi_{n}}} \gamma^{-k}\right\}
\end{aligned}
$$

where

$$
\gamma=\left|z-z^{\prime}\right|+j \rho .
$$

The weights $\eta_{n}^{(l)}$ obtained in this way, although different, are asymptotically equivalent to those in (15). In the new WA method [22], the same remainder expansion is used. But now, instead of acting always on two consecutive partial integrals $T_{\nu n}$, the new WA method acts simultaneously on $n$ members 
of the sequence in (7). Indeed, the system of equations obtained by writing (18) for $n$ different values of $\xi_{i}$ is solved using Cramer's rule, yielding after some algebraic manipulations to the following final expression for the best possible estimation $T_{\nu n}^{*}$ that can be found, out of the $T_{\nu_{i}}$, according the new WA method [22]

$$
T_{\nu n}^{*}=\frac{\sum_{i=1}^{n} \mathrm{w}_{i} T_{\nu_{i}}}{\sum_{i=1}^{n} \mathrm{w}_{i}}
$$

where weights $\mathrm{w}_{i}$ are given as

$$
\mathrm{w}_{i}=(-1)^{i+1}\left(\begin{array}{c}
n-1 \\
i-1
\end{array}\right) e^{\gamma \xi_{i}} \xi_{i}^{n-2-q} .
$$

Equidistant $\xi_{i}$ separated by $\pi / \rho$ and $\pi /\left|z-z^{\prime}\right|$ should be used for $\rho \neq 0$ and $\rho=0$ cases, respectively.

This approach was suggested already in [21] as very powerful from the theoretical point of view. For a long time it was considered inefficient for practical purposes, since it calls for computation of the determinants, which is usually a cumbersome and time-consuming task. Then in [22] it was noticed that those determinants, in the case of SIs, are indeed Vandermonde's type determinants, for which an analytical solution exists.

\section{B. DE Technique}

Based on the original study of Ogata and Sugihara [28], double exponential quadrature formulas, specially tailored for the SI tails of our interest, are developed in [18]. For the sake of internal coherence, the main developments are briefly recalled here. The main idea of the method is as follows. By applying the variable transformation

$$
k_{\rho} \rho=\Phi(t)
$$

where

$$
\begin{aligned}
\Phi(t)= & \frac{\pi}{h} t \cdot \tanh \left(\frac{\pi}{2} \sinh (t)\right) \\
& +\xi_{0} \rho \operatorname{sech}\left(\frac{\pi}{2} \sinh (t)\right) \\
= & \Phi_{1}(t)+\xi_{0} \rho \Phi_{2}(t)
\end{aligned}
$$

the nodes of the final quadrature formulas approach double exponentially to the zeros of the corresponding Bessel function. Therefore, the accurate calculation of the integral (5) can be done by using a moderate number of function evaluations. In (23), $h$ is the parameter of the algorithm, called step size, which directly influences its performance, and therefore has to be carefully chosen.

For the sake of brevity, we show here only the final quadrature formulas for the SI tails, for the two most used indexes, $\nu=0,1$ of the Bessel functions

$$
T_{0} \approx h \sum_{k=1}^{N} w_{0 k} \mathcal{F}_{0}\left(\Phi\left(h \frac{j_{0 k}}{\pi}\right)\right) \Phi^{\prime}\left(h \frac{j_{0 k}}{\pi}\right)
$$

and

$$
\begin{aligned}
T_{1} \approx h \sum_{k=1}^{N} w_{1 k} \mathcal{F}_{1}\left(\Phi\left(h \frac{j_{1 k}}{\pi}\right)\right) \Phi^{\prime}\left(h \frac{j_{1 k}}{\pi}\right) \\
+\left(2 h-\frac{1}{2} \xi_{0} \rho h^{2}\right) \mathcal{F}_{1}\left(\xi_{0} \rho\right)
\end{aligned}
$$

In (24) and (25), $j_{\nu k}$ is the $k$-th zero of the Bessel function $\mathrm{J}_{\nu}$, and the weights $w_{\nu k}$ can be calculated as

$$
w_{\nu k}=\frac{\mathrm{Y}_{\nu}\left(j_{\nu k}\right)}{\mathrm{J}_{\nu+1}\left(j_{\nu k}\right)}=\frac{2}{\pi \xi_{\nu k} \mathrm{~J}_{\nu+1}^{2}\left(\xi_{\nu k}\right)} .
$$

Moreover, function $\mathcal{F}_{\nu}(t)$ is given as follows:

$$
\mathcal{F}_{\nu}(t)=\frac{1}{\rho^{2}} t \tilde{G}\left(\frac{1}{\rho} t ; z \mid z^{\prime}\right) \mathrm{J}_{\nu}(t)
$$

\section{NUMERICAL RESULTS}

In this section we present various numerical results in order to compare accuracy and efficiency of the recently developed methods for the computation of SI tails, discussed in Section II. From the WA family, we have used the Mosig-Michalski transformation [13] known to be the most efficient version. Moreover, the asymptotic evaluation of remainder estimates (16) is used, since it was shown to lead to faster convergence [13]. All the methods were implemented in MATLAB programming language, and all the calculations are done in double precision arithmetic.

First, we consider the family of SIs for which the exact solutions are known thanks to the well-known Sommerfeld identity

$$
\int_{0}^{\infty} \frac{e^{-j k_{z}|z|}}{j k_{z}} \mathrm{~J}_{0}\left(k_{\rho} \rho\right) k_{\rho} d k_{\rho}=\frac{e^{-j k r}}{r}
$$

where $k_{z}=\sqrt{k^{2}-k_{\rho}^{2}}$. Our test cases also include the $\rho$ derivative of (28)

$$
\int_{0}^{\infty} \frac{e^{-j k_{z}|z|}}{j k_{z}} \mathrm{~J}_{1}\left(k_{\rho} \rho\right) k_{\rho}^{2} d k_{\rho}=\rho e^{-j k r} \frac{1+j k r}{r^{3}}
$$

its $z$-derivative:

$$
\int_{0}^{\infty} e^{-j k_{z}|z|} \mathrm{J}_{0}\left(k_{\rho} \rho\right) k_{\rho} d k_{\rho}=z e^{-j k r} \frac{1+j k r}{r^{3}}
$$

as well as its the second derivative with respect to $\rho$ and $z$

$$
\int_{0}^{\infty} e^{-j k_{z}|z|} \mathrm{J}_{1}\left(k_{\rho} \rho\right) k_{\rho}^{2} d k_{\rho}=z \rho e^{-j k r} \frac{3+3 j k r-r^{2} k^{2}}{r^{5}} .
$$

Integrals on the left-hand side of (28)-(31) are split into two parts, as explained in Section II. The first parts of the integrals are evaluated close to machine precision [29], [30]. Therefore, when assessing precision of the methods, we can consider that all the error in computation of the integrals (28)-(31) comes from the evaluation of the SI tail. 
Step size $h$ in (24)-(25) is the key parameter of the DE algorithm since it directly influences the behavior of the algorithm. Hence, its proper choice is very important. While, for the general semi-infinite integrals involving Bessel function kernels the appropriate value of $h$ depends on the problem at hand [28], in [18] it was experimentally found that $h=1 / 32$ stands as the best trade-off between accuracy and computational cost for SI tails. Considering the partition-extrapolation method involving WA extrapolation techniques, the partial integrals $u_{n_{i}}$ in (6) are computed using Gauss-Legendre quadrature of order 16, as suggested in [29].

First we consider the most challenging case, $z=0$, in which the exponential function vanishes, and consequently the integrands in (28)-(31) have the slowest decay. Since the accuracy achieved after certain number of integration points is a good indicator of the efficiency of the method, we plot in Fig. 3 the number of significant digits obtained by using 160 integration points (ten integration intervals for WA) as a function of $k_{0} \rho$. The number of significant digits is computed as $-\log _{10} \mid$ relative error $\mid$. From the presented results, one can see that for the first three examples, all the methods provide very accurate results, with more than 10 exact digits for all the values of $\rho$ studied. However, for (31), the accuracy of the new WA method is significantly higher than that obtained by using the other two methods. The poorest performance is provided by the DE method, which yields to only 7 significant digits accuracy for some values of $\rho$.

We have also considered the behavior of the DE, new WA and Mosig-Michalski WA methods, when applied to SIs (28)-(31), for a wide range of the distances between the source and observation points, $-3 \leq \log _{10}\left(k_{0} \rho\right), \log _{10}\left(k_{0} z\right) \leq 1$. The obtained results are presented in Figs. 4-7. Again, the best accuracy is achieved by using the new WA technique. But, the other two methods lead to very good results as well: more than 8 significant digits accuracy for all the distances considered.

Finally, as a real-life example, we have considered a fourlayer geometry:

- layer-0: PEC;

- layer-1: $\epsilon_{r 1}=4, d_{1}=0.1 \mathrm{~cm}$;

- layer-2: $\epsilon_{r 2}=12.6, d_{2}=0.1 \mathrm{~cm}$;

- layer-3: free-space.

with the source (HED) placed at the interface between the dielectric stack and the free-space. Fig. 8 depicts the scalar potential GF $G_{x}^{q}$ and the component $G_{x x}^{A}$ of the vector potential dyadic GF, when the source and the observer belong to the same plane $\left(z=z^{\prime}=0\right)$ at $f=30 \mathrm{GHz}$. There is a perfect agreement between the three techniques with the curves on top of each other for the full range of distances. Furthermore, since the analytical solution does not exist in this case, in order to asses the accuracy of the obtained results, we took as reference the results obtained applying the Mosig-Michalski algorithm until machine precision was reached. The results obtained using the other two methods were then compared with the reference result. Fig. 9 gives the number of significant digits of relative error. The standard number of 160 points has been used. The three methods agree in more than 11 significant digits.

A final word must be said on the computational time and on the efficient implementation of the above described methods

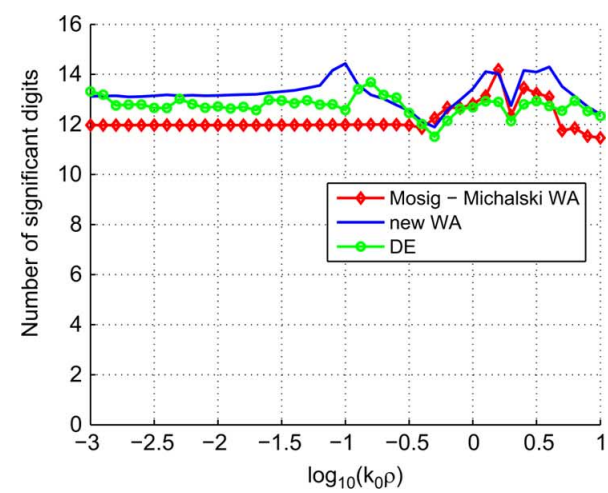

(a)

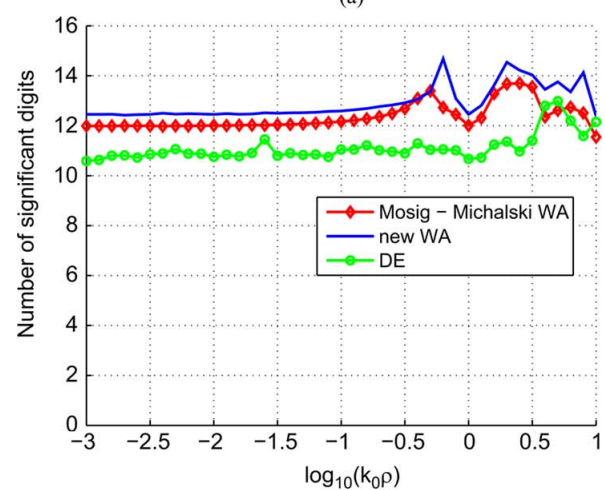

(b)

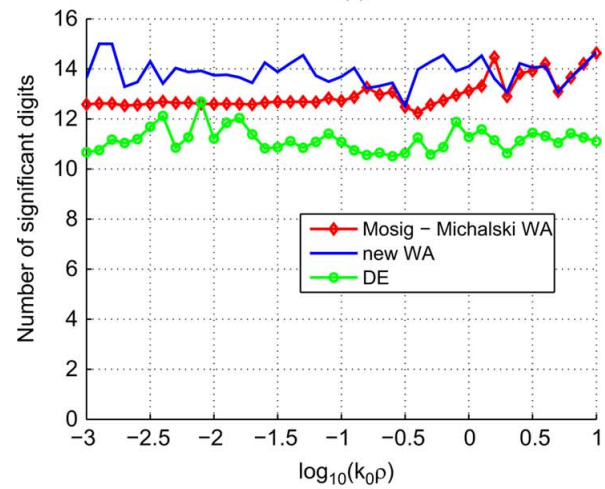

(c)

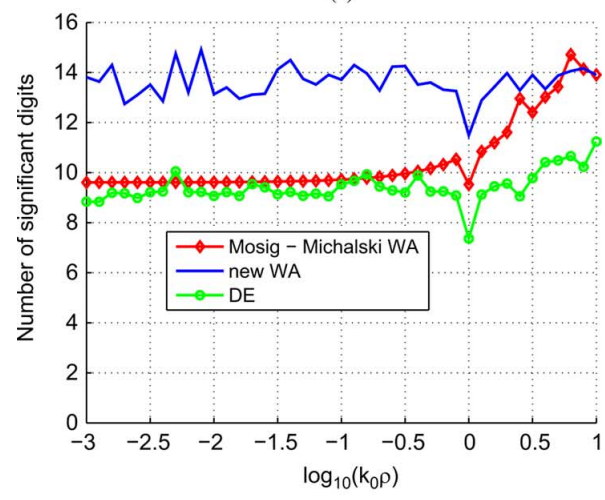

(d)

Fig. 3. Number of significant digits obtained by using 160 integration points (a) Sommerfeld identity tail (28) (b) $\rho$-derivative of the Sommerfeld identity tail (29) (c) $z$-derivative of the Sommerfeld identity tail (30) (d) Second derivative of the Sommerfeld identity tail with respect to $\rho$ and $z$ (31).

for numerical evaluation of SI tails. Since the value of the step-size parameter $h$ of DE quadrature is chosen a priori, the 


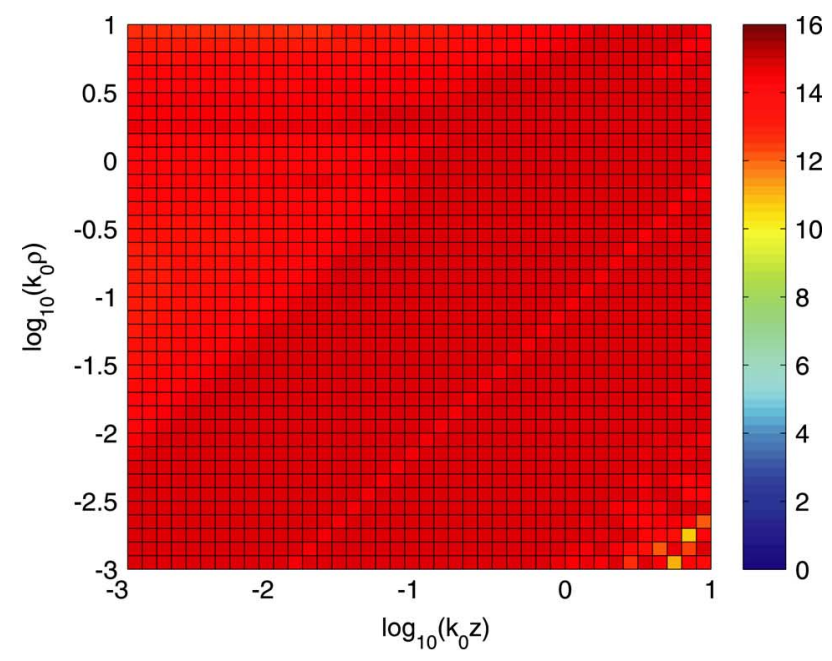

(a)

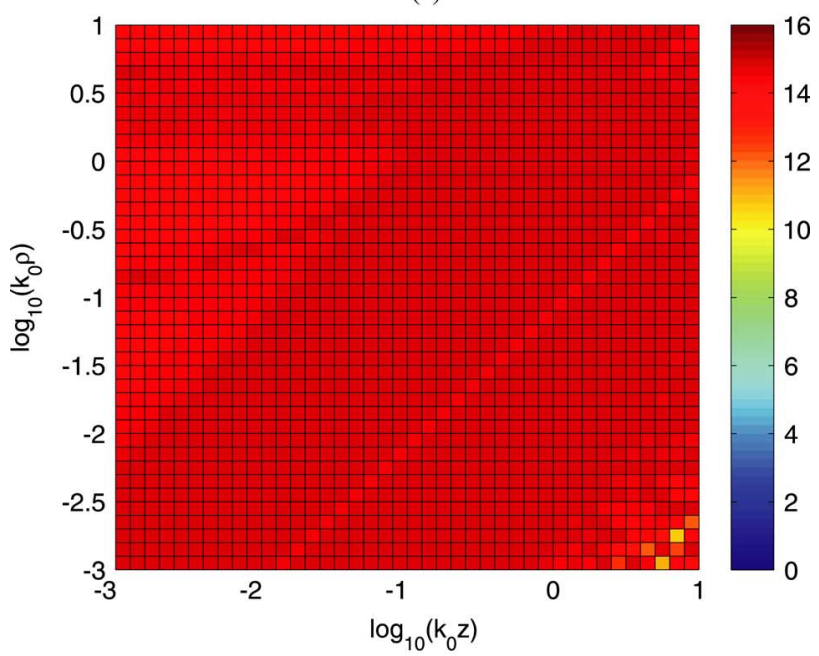

(b)

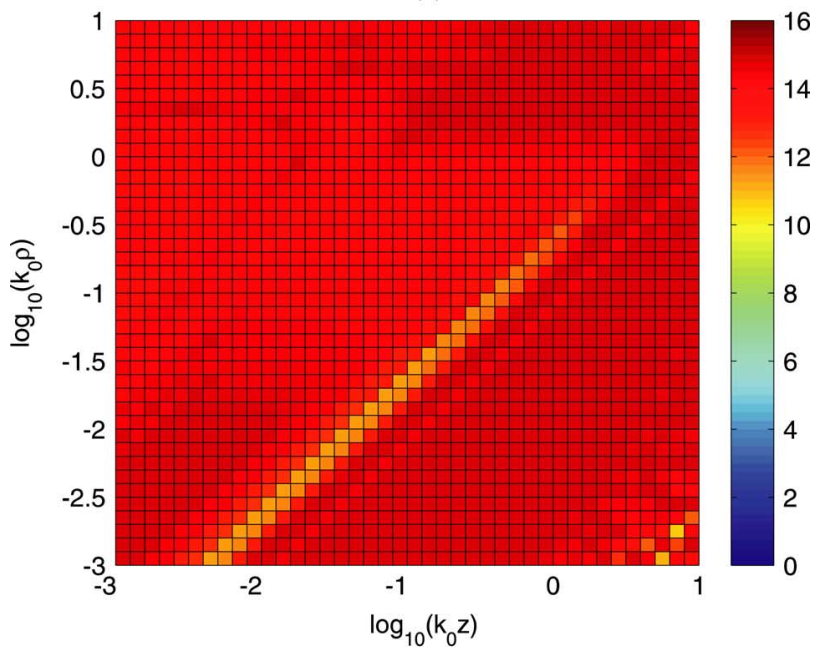

(c)

Fig. 4. Number of significant digits for the SI (28), obtained by using 160 integration points, for a wide range of distances from the source (a) MosigMichalski transformation (b) New WA algorithm (c) DE algorithm.

associated weights and abscissas can be precomputed. Therefore, the direct application of the formulas (24)-(25) using as default the maximal number of points (160, corresponding to

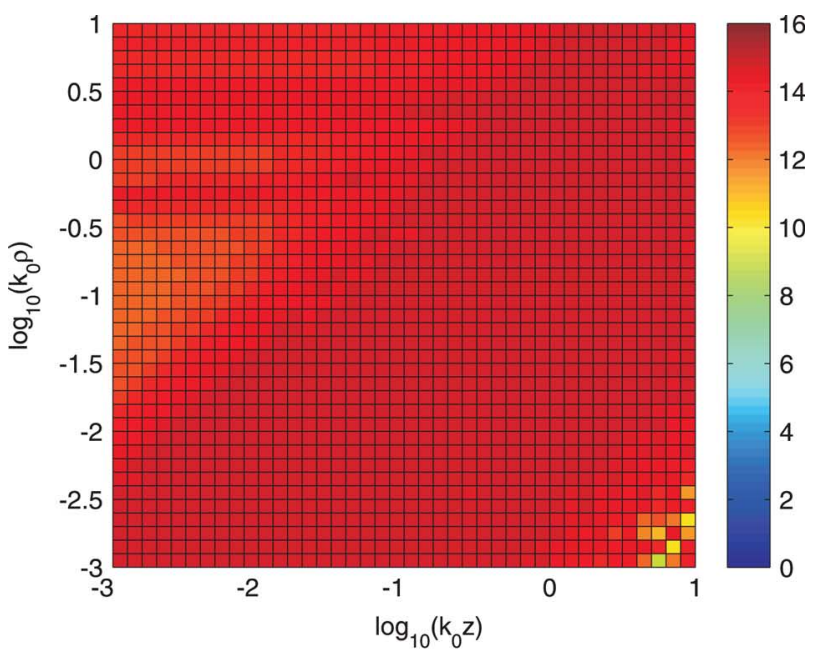

(a)

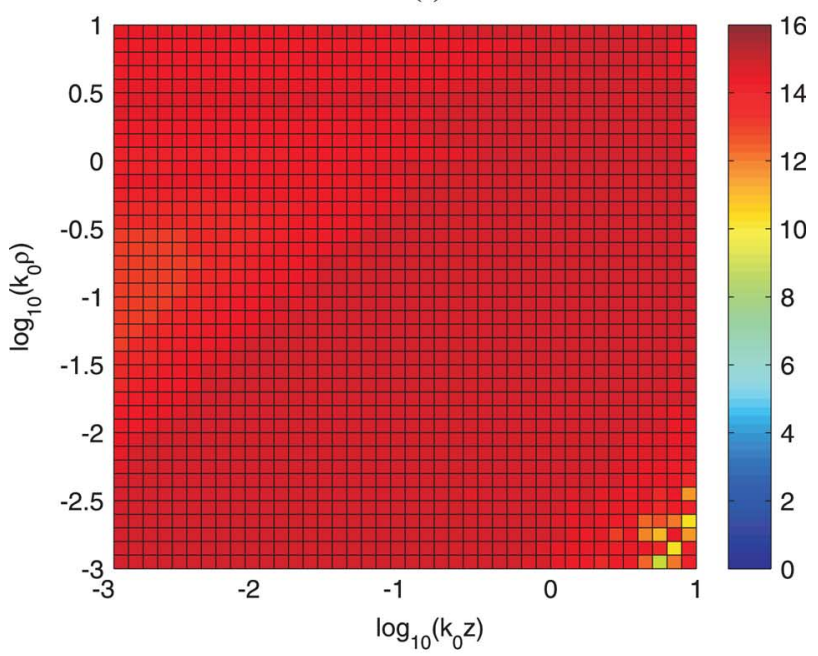

(b)

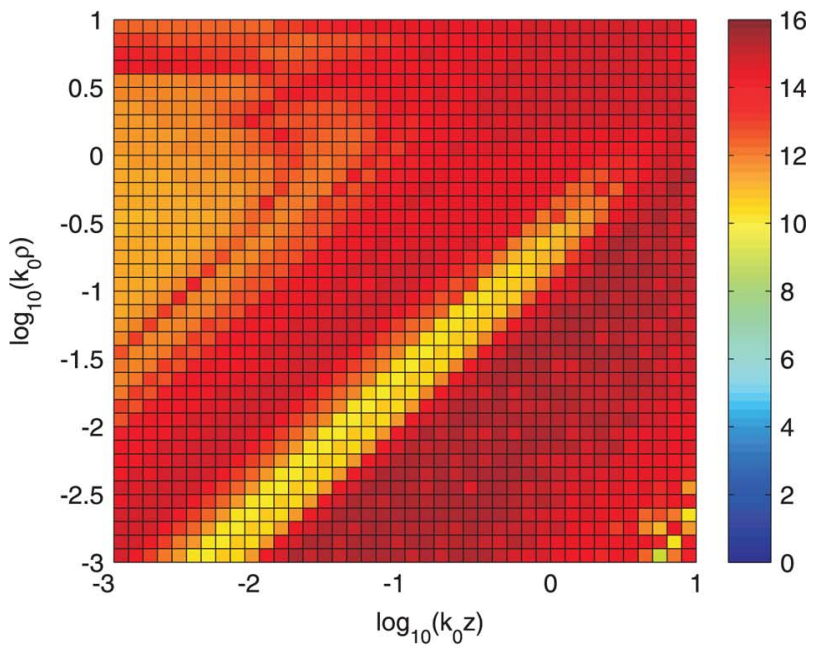

(c)

Fig. 5. Number of significant digits for the SI (29), obtained by using 160 integration points, for a wide range of distances from the source (a) MosigMichalski transformation (b) New WA algorithm (c) DE algorithm.

10 iterations with a quadrature order of 16) can be implemented in MATLAB as a simple matrix multiplication, which is very convenient and indeed leads to lower computational time than 


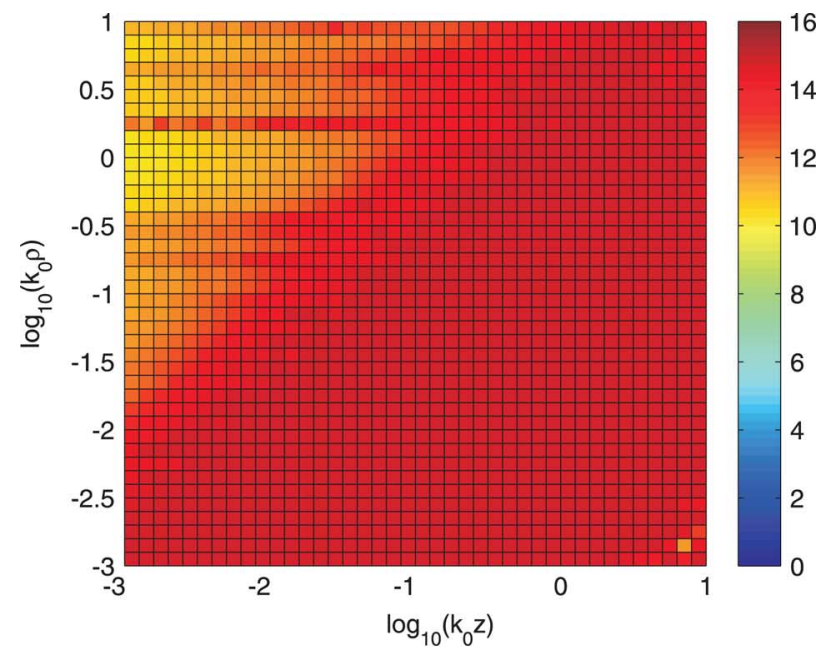

(a)

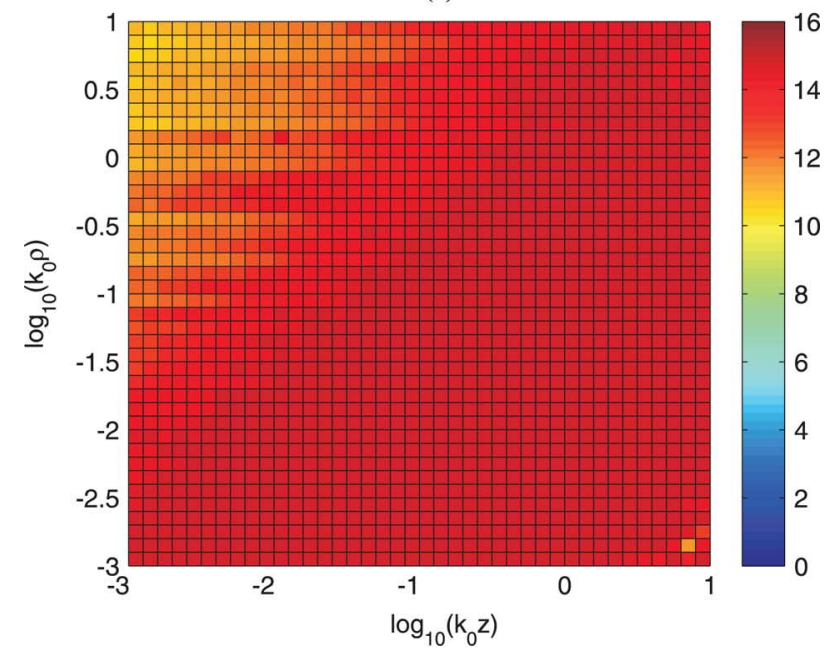

(b)

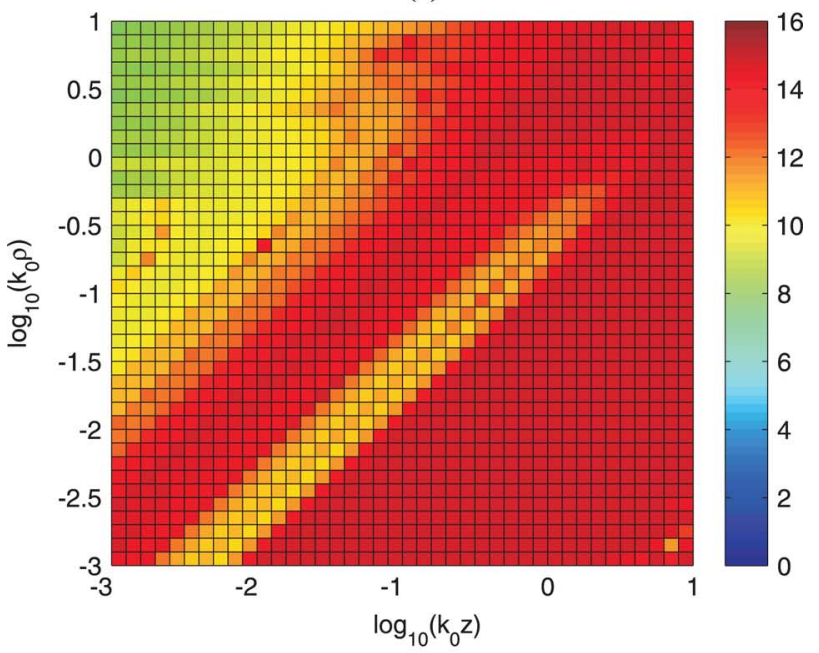

(c)

Fig. 6. Number of significant digits for the SI (30), obtained by using 160 integration points, for a wide range of distances from the source (a) MosigMichalski transformation (b) New WA algorithm (c) DE algorithm.

the iterative procedure proposed in [18], while resulting in the same accuracy for the final results. Under these assumptions, the DE transformation needs, approximately 5 times less computational time than the two other methods, as can be seen from

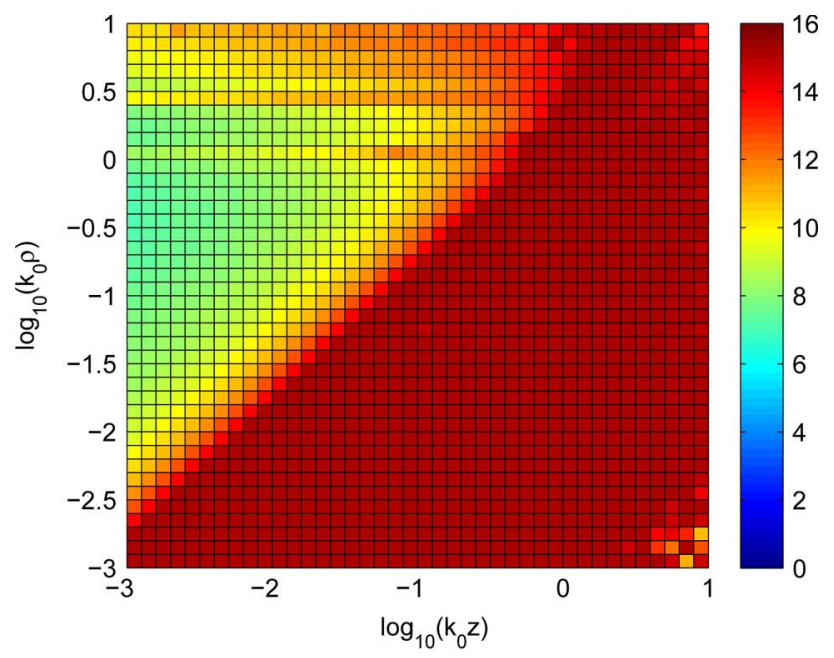

(a)

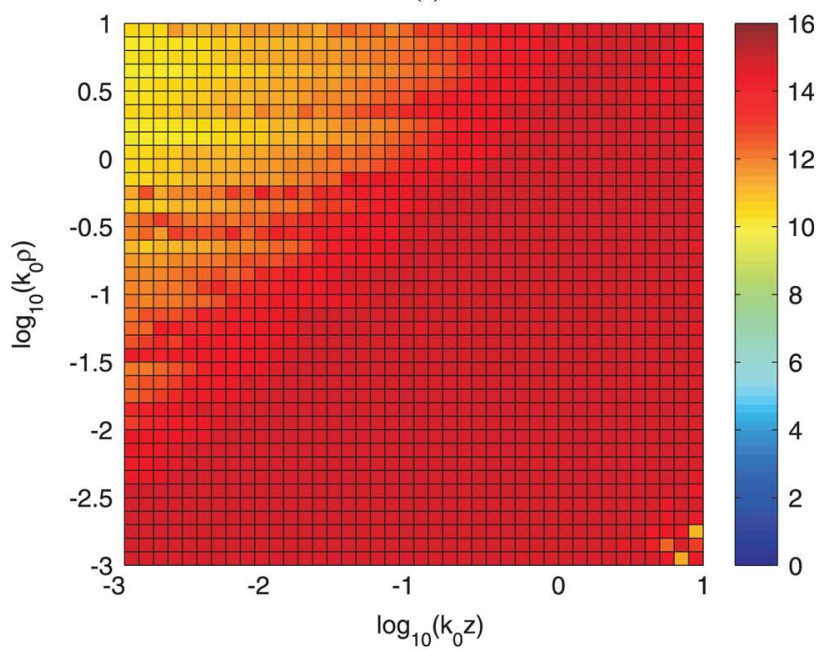

(b)

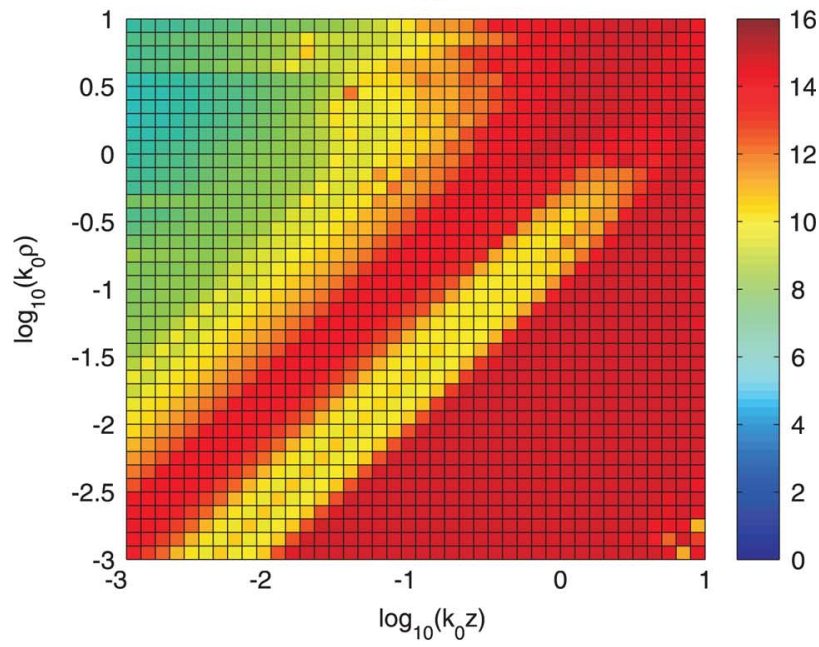

(c)

Fig. 7. Number of significant digits for the SI (31), obtained by using 160 integration points, for a wide range of distances from the source (a) MosigMichalski transformation (b) new WA algorithm (c) DE algorithm.

Fig. 10. In the WA technique, the main part of the computation time is consumed by the evaluation of the 10 partial integrals. The overhead associated to the WA algorithms themselves is very reduced, which makes the total time almost the same in 


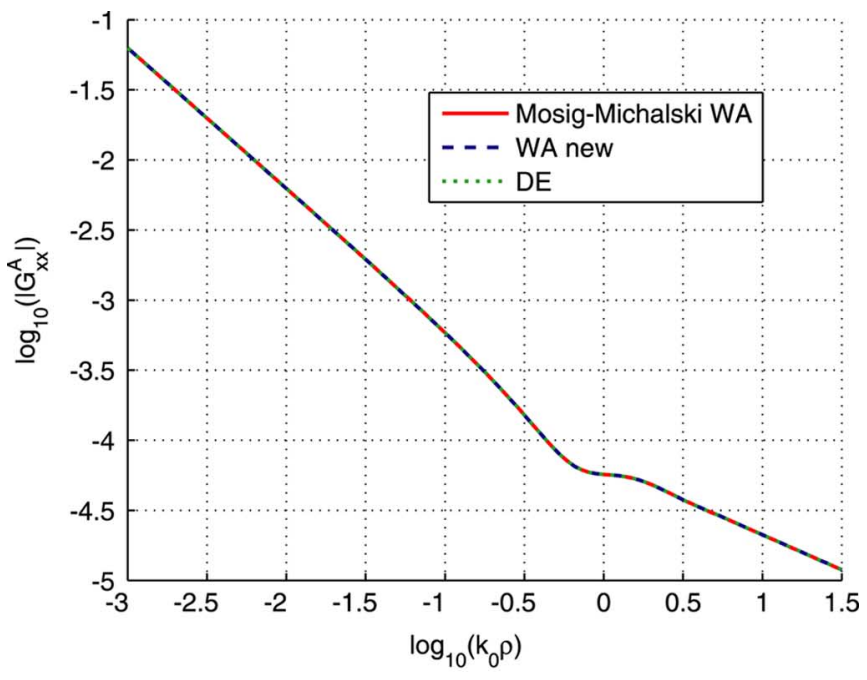

(a)

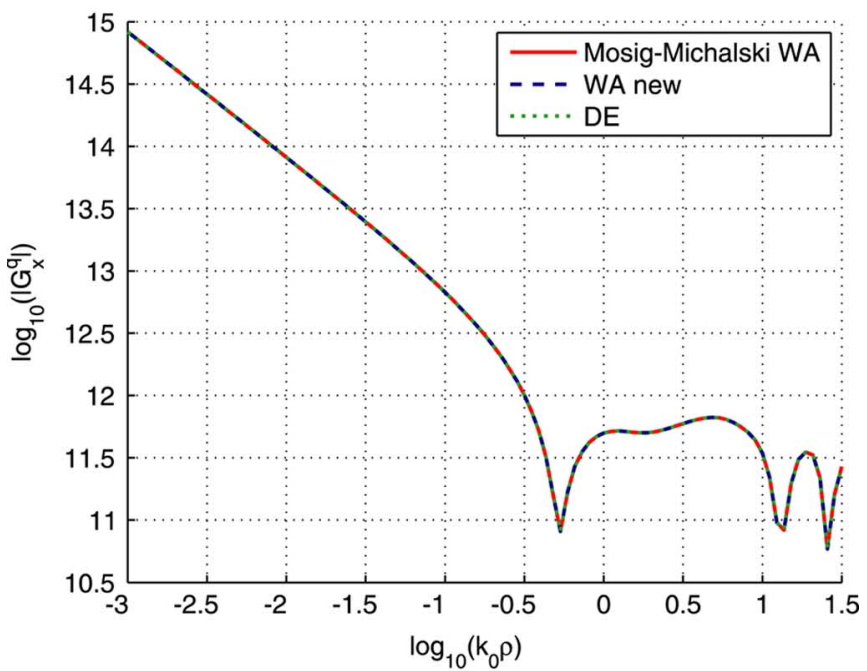

(b)

Fig. 8. Spatial domain Green's function of four layer media problem at $30 \mathrm{GHz}$ (a) $G_{x x}^{A}\left(\rho ; z, z^{\prime}\right)$ (b) $G_{x}^{q}\left(\rho ; z, z^{\prime}\right)$.

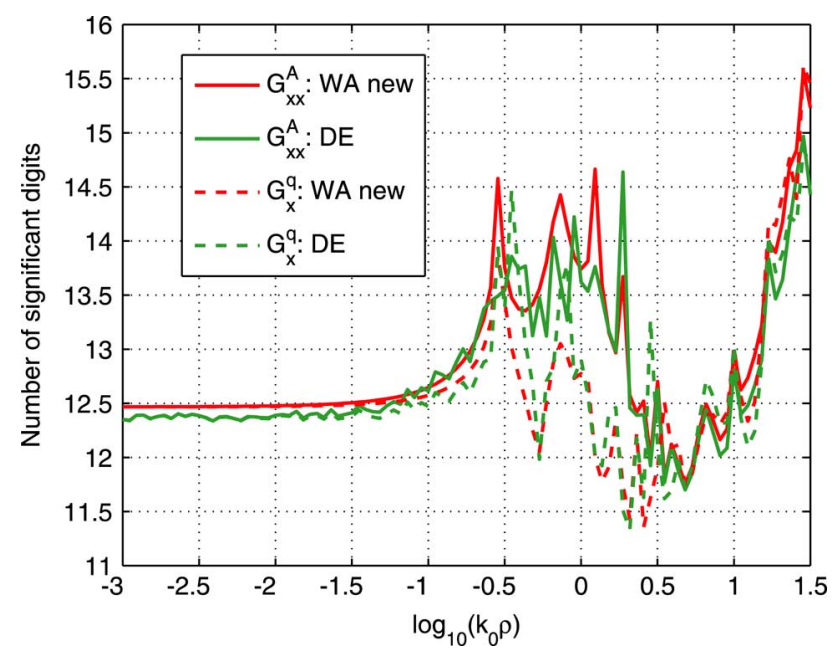

Fig. 9. Number of significant digits of relative error for four-layer geometry at $30 \mathrm{GHz}$.

both cases (Mosig-Michalski and new WA). On the other hand, $\mathrm{DE}$ is noticeably ( $\sim 5$ times) faster for the same SI tail. The

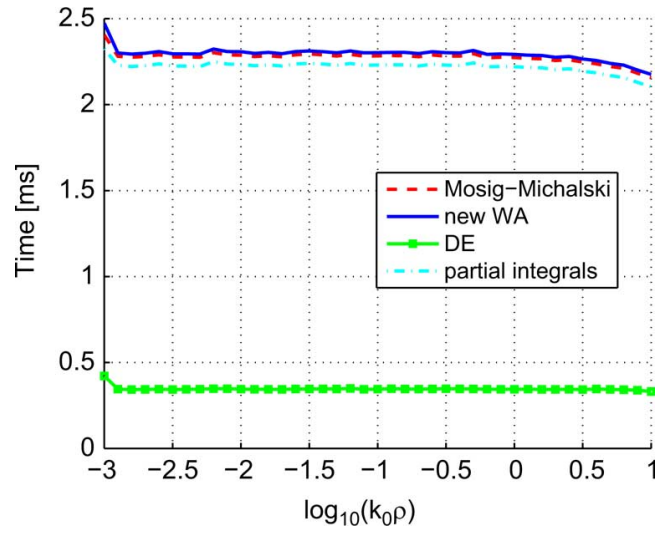

Fig. 10. Time comparison for the SI tail (28).

weights (15)-(16) involved in the Mosig-Michalski WA technique are very easy to evaluate, but the method calls for the recursion (17). On the other hand, the evaluation of the weights (21) involved in new WA algorithm is more complex, since it calls for the computation of the binomial coefficients. However, this is not a problem since many programming languages have built-in functions for the evaluation of binomial coefficients. Alternatively, an iterative procedure might be used. Roughly, this amounts to say that the overhead of the Mosig-Michalski WA transformation is time-wise equivalent to the evaluation of more complicated weights in the new WA method. However the dominant part of computational time goes to the accurate evaluation of the sequence of partial sums, $T_{\nu i}$.

\section{CONCLUSION}

In this paper we have discussed two recently developed techniques for the efficient numerical evaluation of SI tails, mainly a new version of the Weighted Averages (WA) algorithm and a specially tailored Double Exponential (DE) quadrature formula. The performances of the methods are compared throughout a series of representative numerical examples, with the target of assessing the advantages and disadvantages of their application. In general, the new WA algorithm is shown to be the winner in terms of accuracy. It converges faster towards the final values, when compared to Mosig-Michalski WA technique. This may be an important asset, as the dominant part of the computational time is usually due to the evaluation of the partial integrals in the sequence. Hence, the new WA can be used advantageously as an alternative to the classic (Mosig-Michalski) WA algorithm, today the most currently used extrapolation method in integration-then-summation techniques. On the contrary, the DE quadrature formula, although it gives in average lower accuracy when compared with WA methods, still yields very good results. More precisely, it always provides more than 9 significant digits of accuracy of relative error, except in the case of (26), when it leads to only 7 digits accuracy for some combination of $\rho$ and $z$ values. However, in the MPIE formulation this type of integrals arises only in the post-processing related to the fields evaluations. Properly implemented, DE always performed significantly faster than both versions of WA (up to 5 times). This is to be considered in applications where speed is the primordial parameter. 


\section{REFERENCES}

[1] J. R. Mosig, "Arbitrary shaped microstrip structures and their analysis with a mixed potential integral equation," IEEE Trans. Microw. Theory Tech., vol. 36, no. 2, pp. 314-323, Feb. 1988

[2] K. A. Michalski, "The mixed-potential electric field integral equation for objects in layered media," Arch. Elek. Ubertragung., vol. 39, no. 5, pp. 317-322, 1985 .

[3] K. A. Michalski and D. Zheng, "Electromagnetic scattering and radiation by surfaces of arbitrary shape in layered media, Part 1: Theory," IEEE Trans. Antennas Propag., vol. 38, no. 3, pp. 335-344, Mar. 1990.

[4] M. I. Aksun and G. Dural, "Clarification of issues on the closed-form Green's functions in stratified media," IEEE Trans. Antennas Propag., vol. 53, no. 11, pp. 3644-3653, Nov. 2005.

[5] M. Yuan, T. K. Sarkar, and M. Salazar-Palma, "A direct discrete complex image method from the closed-form Green's functions in multilayered media," IEEE Trans. Microw. Theory Tech., vol. 54, no. 3, pp. 1025-1032, Mar. 2006

[6] V. N. Kourkoulos and A. C. Cangellaris, "Accurate approximation of Green's functions in planar stratified media in terms of a finite sum of spherical and cylindrical waves," IEEE Trans. Antennas Propag., vol. 54, no. 5, pp. 1568-1576, May 2006

[7] R. R. Boix, F. Mesa, and F. Medina, "Application of total least squares to the derivation of closed-form Green's functions for planar layered media," IEEE Trans. Microw. Theory Tech., vol. 55, no. 2, pp. 268-280, Feb. 2007.

[8] A. G. Polimeridis, T. V. Yioultsis, and T. D. Tsiboukis, "A robust method for the computation of Green's functions in stratified media," IEEE Trans. Antennas Propag., vol. 55, no. 7, pp. 1963-1969, Jul. 2007.

[9] A. G. Polimeridis, T. V. Yioultsis, and T. D. Tsiboukis, "Fast numerical computation of Green's functions for unbounded planar stratified media with a finite-difference technique and Gaussian spectral rules,' IEEE Trans. Microw. Theory Tech., vol. 55, no. 1, pp. 100-107, Jan. 2007.

[10] A. G. Polimeridis and T. V. Yioultsis, "On the efficient computation of closed-form Green's functions in planar stratified media," Int. J. RF and Microw. CAE, vol. 18, no. 2, pp. 118-126, 2008.

[11] A. Alparslan, M. I. Aksun, and K. A. Michalski, "Closed-form Green's functions in planar layered media for all ranges and materials," IEEE Trans. Microw. Theory Tech., vol. 58, no. 3, pp. 602-613, Mar. 2010.

[12] N. Kinayman and M. I. Aksun, "Comparative study of acceleration techniques for integrals and series in electromagnetic problems," Radio Sci., vol. 30, no. 6, pp. 1713-1722, 1995

[13] K. A. Michalski, "Extrapolation methods for Sommerfeld integral tails," IEEE Trans. Antennas Propag., vol. 46, no. 10, pp. 1405-1418, Oct. 1998.

[14] T. Hasegawa and A. Sidi, "An automatic integration procedure for infinite range integrals involving oscillatory kernels," Numerical Algorithms, vol. 13 , no. 1, pp. 1-19, 1996.

[15] T. Ooura, "A continuous Euler transformation and its application to the Fourier transforms of a slowly decaying functions," J. Comput. Appl. Math., vol. 130, no. 1-2, pp. 259-270, 2001.

[16] S. Singh and R. Singh, "Computation of Sommerfeld integrals using tanh transformation," MOTL, vol. 37, no. 3, pp. 177-180, 2003.

[17] T. Ooura, "A generalization of the continuous Euler transformation and its application to numerical quadrature," J. Comput. Appl. Math., vol. 157 , no. 2, pp. 251-259, 2003.

[18] R. Golubovic-Niciforovic, A. Polimeridis, and J. R. Mosig, "Fast computation of Sommerfeld integral tails via direct integration based on the double exponential-type quadrature formulas," IEEE Trans. Antennas Propag., vol. 59, no. 2, pp. 694-699, Feb. 2010.

[19] J. R. Mosig and F. E. Gardiol, "A dynamical radiation model for microstrip structures," in Advances in Electronics and Electron Physics. New York: Academic, 1982, pp. 139-237.

[20] J. R. Mosig and F. E. Gardiol, "Analytical and numerical techniques in the Green's function treatment of microstrip antennas and scatterers," Proc. Inst. Elect. Eng. Part H-Microwaves, Optics and Antennas, vol. 130, no. 2, pp. 175-182, 1983.

[21] J. R. Mosig, "Integral equation techniques," in Numerical Techniques for Microwave and Milimeter-Wave Passive Structures, T. Itoh, Ed. New York: Wiley, 1989, pp. 133-213.

[22] J. R. Mosig, "The weighted averages algorithm revisited," IEEE Trans. Antennas Propag. pp. 1-9 [Online]. Available: http://ieeexplore.ieee.org

[23] H. Takahasi and M. Mori, "Double exponential formulas for numerical integration," Publ. RIMS, Kyoto Univ., vol. 9, no. 3, pp. 721-741, 1974.

[24] T. Ooura and M. Mori, "A robust double exponential formula for Fourier-type integrals," J. Comput. Appl. Math., vol. 112, no. 1-2, pp. 229-241, 1999.
[25] C. Frappier and P. Olivier, "A quadrature formula involving zeros of Bessel functions," Math. Comp., vol. 60, no. 201, pp. 303-316, 1993.

[26] G. R. Grozev and Q. I. Rahman, "Quadrature formula with zeros of Bessel functions as nodes," Math. Comp., vol. 64, no. 210, pp. 715-725, 1995.

[27] R. B. Ghanem, "Quadrature formulae using zeros of Bessel function as nodes," Math. Comp., vol. 67, no. 221, pp. 323-336, 1998

[28] H. Ogata and M. Sugihara, "Quadrature formulae for oscillatory infinite integrals involving the Bessel functions (in Japanese)," Trans. Japan Soc. Ind. Appl. Math., vol. 8, no. 2, pp. 223-256, 1998.

[29] K. A. Michalski, "Application of the complex image method to electromagnetic field computation in planar uniaxial multilayers,' presented at the Workshop Integral Techniques for Electromagnetics (INTELECT), Lausanne, Switzerland, 2007.

[30] P. Gay-Balmaz and J. R. Mosig, "Three-dimensional planar radiating structures in stratified media," Int. J. RF and Microw. CAE, vol. 7, no. 5, pp. 330-343, 1997.

[31] T. N. L. Patterson, "Algorithm 468: Algorithm for automatic numerical integration over a finite interval," Commun. $A C M$, vol. 16, no. 11, pp. 694-699, 1973.

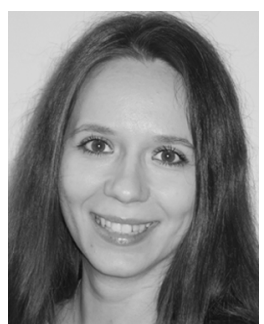

Ruzica Golubovic was born in Belgrade, Serbia, in 1983. She received the Dipl.Ing. degree from the School of Electrical Engineering (ETF), University of Belgrade, in 2006 and the Ph.D. degree from the Ecole Polytechnique Fédérale de Lausanne (EPFL), Lausanne, Switzerland, in 2011

Her research interests include computational electromagnetics, with emphasis on planar multilayered media and optimization techniques.

Athanasios G. Polimeridis was born in Thessaloniki, Hellas, in 1980. He received the Diploma degree in electrical engineering and the Ph.D. degree from the Department of Electrical and Computer Engineering, Aristotle University of Thessaloniki (AUTH), Hellas, in 2003 and 2008, respectively.

Since October 2008, he has been a Postdoctoral Research Associate with the Laboratory of Electromagnetics and Acoustics, Swiss Federal Institute of Technology (Ecole Polytechnique Fédérale de Lausanne, EPFL), Switzerland. His research interests lie in the broad area of computational electromagnetics, and revolve around the development and implementation of integral-equation based algorithms.

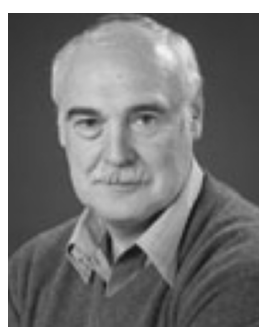

Juan R. Mosig (S'76-M'87-SM'94-F'99) was born in Cádiz, Spain. He received the Electrical Engineer degree from the Universidad Politécnica de Madrid, Madrid, Spain, in 1973, and the Ph.D. degree from the Ecole Polytechnique Fédérale de Lausanne (EPFL), Lausanne, Switzerland, in 1983.

Since 1991, he has been a Professor at EPFL and since 2000, he has been the Head of the Laboratory of Electromagnetics and Acoustics (LEMA) at EPFL. In 1984, he was a Visiting Research Associate with the Rochester Institute of Technology, Rochester, NY, and Syracuse University, Syracuse, NY. He has also held scientific appointments with the University of Rennes, France, the University of Nice, France, the Technical University of Denmark at Lyngby and the University of Colorado at Boulder. At EPFL, he is currently a co-Director of the College of Humanities and the Chairman of the EPFL Space Center, conducting many Swiss research projects for the European Space Agency (ESA). He has authored five chapters in books on planar antennas and circuits and over 100 peer-reviewed journal papers. His current research interests include electromagnetic theory, numerical methods, and planar antennas.

Dr. Mosig is a Fellow of the IEEE, the Chairperson of the European COST Action on Antennas "ASSIST" (2007-2011) and a Founding Member and Acting Chair of the European Association \& Conferences on Antennas and Propagation (EurAAP and EuCAP). 